\title{
Correlation of preoperative MRI with the long-term outcomes of dorsal root entry zone lesioning for brachial plexus avulsion pain
}

\author{
Andrew L. Ko, MD, ${ }^{1}$ Alp Ozpinar, MD, ${ }^{2}$ Jeffrey S. Raskin, MD, ${ }^{2}$ Stephen T. Magill, MD, PhD, ${ }^{3}$ \\ Ahmed M. Raslan, MD, ${ }^{2}$ and Kim J. Burchiel, MD² \\ 'Department of Neurological Surgery, University of Washington, Seattle, Washington; 2Department of Neurological Surgery, \\ Oregon Health \& Science University, Portland, Oregon; and 'Department of Neurological Surgery, University of California, \\ San Francisco, California
}

OBJECTIVE Lesioning of the dorsal root entry zone (DREZotomy) is an effective treatment for brachial plexus avulsion (BPA) pain. The role of preoperative assessment with MRI has been shown to be unreliable for determining affected levels; however, it may have a role in predicting pain outcomes. Here, DREZotomy outcomes are reviewed and preoperative $\mathrm{MRI}$ is examined as a possible prognostic factor.

METHODS A retrospective review was performed of an institutional database of patients who had undergone brachial plexus DREZ procedures since 1995. Preoperative MRI was examined to assess damage to the DREZ or dorsal horn, as evidenced by avulsion of the DREZ or T2 hyperintensity within the spinal cord. Phone interviews were conducted to assess the long-term pain outcomes.

RESULTS Between 1995 and 2012, 27 patients were found to have undergone cervical DREZ procedures for BPA. Of these, 15 had preoperative MR images of the cervical spine available for review. The outcomes were graded from 1 to 4 as poor (no significant relief), good (more than $50 \%$ pain relief), excellent (more than $75 \%$ pain relief), or pain free, respectively. Overall, DREZotomy was found to be a safe, efficacious, and durable procedure for relief of pain due to BPA. The initial success rate was $73 \%$, which declined to $66 \%$ at a median follow-up time of 62.5 months. Damage to the DREZ or dorsal horn was significantly correlated with poorer outcomes $(p=0.02)$. The average outcomes in patients without MRI evidence of DREZ or dorsal horn damage was significantly higher than in patients with such damage (3.67 vs 1.75 , t-test; $p=0.001)$. A longer duration of pain prior to operation was also a significant predictor of treatment success $(p=0.004)$.

CONCLUSIONS Overall, the DREZotomy procedure has a $66 \%$ chance of achieving meaningful pain relief on longterm follow-up. Successful pain relief is associated with the lack of damage to the DREZ and dorsal horn on preoperative MRI.

http://thejns.org/doi/abs/10.3171/2015.2.JNS142572

KEY WORDS brachial plexus avulsion; neuropathic pain; DREZotomy; dorsal root entry zone

$\mathrm{B}$ RACHIAL plexus avulsion (BPA) injuries occur in about $1 \%$ of multitrauma victims, predominantly in young males between 18 and 25 years of age. ${ }^{18,20}$ These injuries are associated with deafferentation pain in up to $90 \%$ of patients. ${ }^{3,3,15,18,20}$ Pain may present immediately after trauma, or months to years later, and does not often remit spontaneously. ${ }^{20}$ The mechanism of pain generation is thought to be the spontaneous and erratic firing of secondary sensory neurons in the dorsal horn as a result of avulsion injury; this hypothesis has been supported by animal and human studies on the spinal cord after deaffer- entation..$^{7,14,26}$ Pain associated with BPA is generally characterized by 2 components: paroxysmal lancinating pain, and continuous burning or throbbing pain. Both components are resistant to all classes of analgesics, antidepressants, and anticonvulsants. ${ }^{20}$

The surgical treatment of chronic pain may be performed via neuromodulation or ablative procedures. Spinal cord and deep brain stimulation have been shown to provide reduction in pain after BPA, as measured by the visual analog scale (VAS), with a statistically significant but slight reduction in pain scores. ${ }^{21,22}$ Ablative procedures

ABBREVIATIONS BPA = brachial plexus avulsion; DREZ = dorsal root entry zone; DZEZotomy = dorsal root entry zone lesioning; VAS = visual analog scale. SUBMITTED November 10, 2014. ACCEPTED February 25, 2015.

INCLUDE WHEN CITING Published online September 25, 2015; DOI: 10.3171/2015.2.JNS142572. 
such as medial thalamotomy, spinothalamic tractotomy, and anterolateral cordotomy have not demonstrated longterm benefits and have disabling side effects. ${ }^{30}$

On the other hand, dorsal root entry zone (DREZ) lesioning-or DREZotomy - has been shown to be a durable and effective treatment for neuropathic pain resulting from BPA. $1,2,5,9,24,25,27,29$ Though not as frequently performed as a few decades ago, DREZotomy remains a potent surgical therapy for the management of pain from BPA and other spinal cord injuries. ${ }^{1,26}$ DREZotomy involves the surgical destruction of the erratically firing second-order neurons of the ascending nociceptive pathway, and clinical outcomes following DREZotomy have been correlated with the abolition of spontaneous and evoked hyperactivity in the dorsal horn.,11,24 The procedure was first described by Sindou in 1972 using an arachnoid knife to incise the spinal cord at the DREZ before enlarging the lesion using thermal coagulation. ${ }^{26}$ Additional techniques using radiofrequency thermablation, ${ }^{3}$ focused laser-beam treatment, ${ }^{13}$ and an ultrasonic microprobe ${ }^{6}$ have been developed with similar results.

The outcomes of DREZotomy for BPA show a more dramatic reduction in pain levels compared with neuromodulatory techniques. Sindou et al. reported that $66 \%$ percent of patients treated with DREZotomy have complete relief or more than $75 \%$ reduction in pain, with $71 \%$ noting improvement in their daily activities. ${ }^{27}$ Other studies have shown similar long-term outcomes ranging from $50 \%$ to $75 \%$ abolition of pain. ${ }^{5,8,25}$

The role of preoperative imaging of the spinal cord for DREZotomy has not been well defined. Studies have shown that MRI has not been a reliable predictor of affected levels. For example, the presence of pseudomeningoceles, while considered a classic indirect sign of root avulsion, is not a sensitive indicator of the injury level, with up to $50 \%$ of partially or totally avulsed roots showing no observable meningeal alterations on MRI. ${ }^{1}$ As a result, determining the levels to treat is a clinical decision made by noting the motor and sensory deficits and directly examining the spinal cord intraoperatively; segments with frankly absent, partially avulsed, grayish, or atrophic-appearing rootlets are treated. ${ }^{27}$

Focal gliosis and microcysts of the DREZ may also be seen at the time of surgery. These injuries to the spinal cord itself can be visualized on preoperative $\mathrm{MRI}^{17}$ and may have prognostic value. If the success of DREZotomy is predicated based on the successful ablation of abnormally hyperactive interneurons within the DREZ, the extent of injury to the spinal cord may impact the success rate of this procedure. In other words, if the DREZ is injured to the point that the secondary nociceptive pathways are already inactive, then the procedure may be less successful. This may be because the pain is not the result of aberrant signaling within the spinal cord, but the result of a more central process. With this in mind, whether the examination of these imaging findings has any prognostic value has yet to be determined.

In this paper, we retrospectively examine the long-term outcomes of performing DREZotomy for BPA in relation to the imaging characteristics of the spinal cord in order to identify factors that predict the outcomes for DREZotomy.

\section{Methods}

\section{Study Design}

This study is a retrospective review of patients 18 years or older who were treated at Oregon Health \& Science University between 1995 and 2012. We identified all patients with upper-extremity neuropathic pain who were treated with cervical DREZotomy. The study was approved by the institutional review board.

Of 29 patients found to have undergone DREZotomy, 1 patient showed no cervical root avulsion at surgery and 1 patient presented with metastatic breast cancer invading the brachial plexus. A total of 27 patients were therefore included in the study. A phone survey, chart review, and imaging review were completed to confirm the diagnosis, assess the demographic and clinical characteristics, and ascertain outcomes. One patient could not be reached for phone survey, and therefore the last clinic visit was used as the follow-up with the data censored accordingly. Telephone surveys were performed by a single interviewer who was not involved in patient care. Preoperative cervical spine MR images were available for 15 patients. Radiological review was performed independently by 2 observers.

\section{Surgical Procedure}

Following endotracheal intubation and administering prophylactic antibiotics, each patient was positioned prone on gel rolls and a Mayfield head clamp was applied. A unilateral subperiosteal paraspinal muscle elevation was performed. The thecal sac was exposed and the desired levels were spanned via hemilaminectomy. The dura was opened. The operating microscope was used for the remainder of the procedure.

Microscopic inspection was performed to confirm root avulsion. The pial vasculature was examined to identify the DREZ. Variable microsurgical techniques were employed for lesion creation, including radiofrequency ablation, bipolar electrocautery, and use of the Beaver knife. In all cases, the desired lesion was elaborated $2 \mathrm{~mm}$ deep and directed at a $45^{\circ}$ angle into the intermediolateral sulcus just ventral to DREZ and Lissauer's tract of the spinal cord (Fig. 1). Lesions were made in a staccato fashion in a manner that minimally disrupted the pia.

Once the DREZotomy was completed, dural and soft tissue closure was performed in the standard fashion.

\section{Outcomes Assessment}

Pain outcomes were assessed from 0 to 10 using the VAS and categorized into 4 groups of pain relief: complete (4), excellent (3), good (2), or poor (1). Patients with complete pain relief no longer have neuropathic pain. Excellent pain relief includes patients with pain reduction of $75 \%$ who no longer require medications. Patients with good results experienced pain reduction of more than $50 \%$, may require medications, and are satisfied with their operative result. Patients with poor pain relief have no significant changes from their preoperative pain levels or consider the operation unsuccessful. Success was defined as a good, excellent, or pain-free outcome. Treatment failure was considered to occur at a point when the patient was no longer in the good, excellent, or pain-free category. 

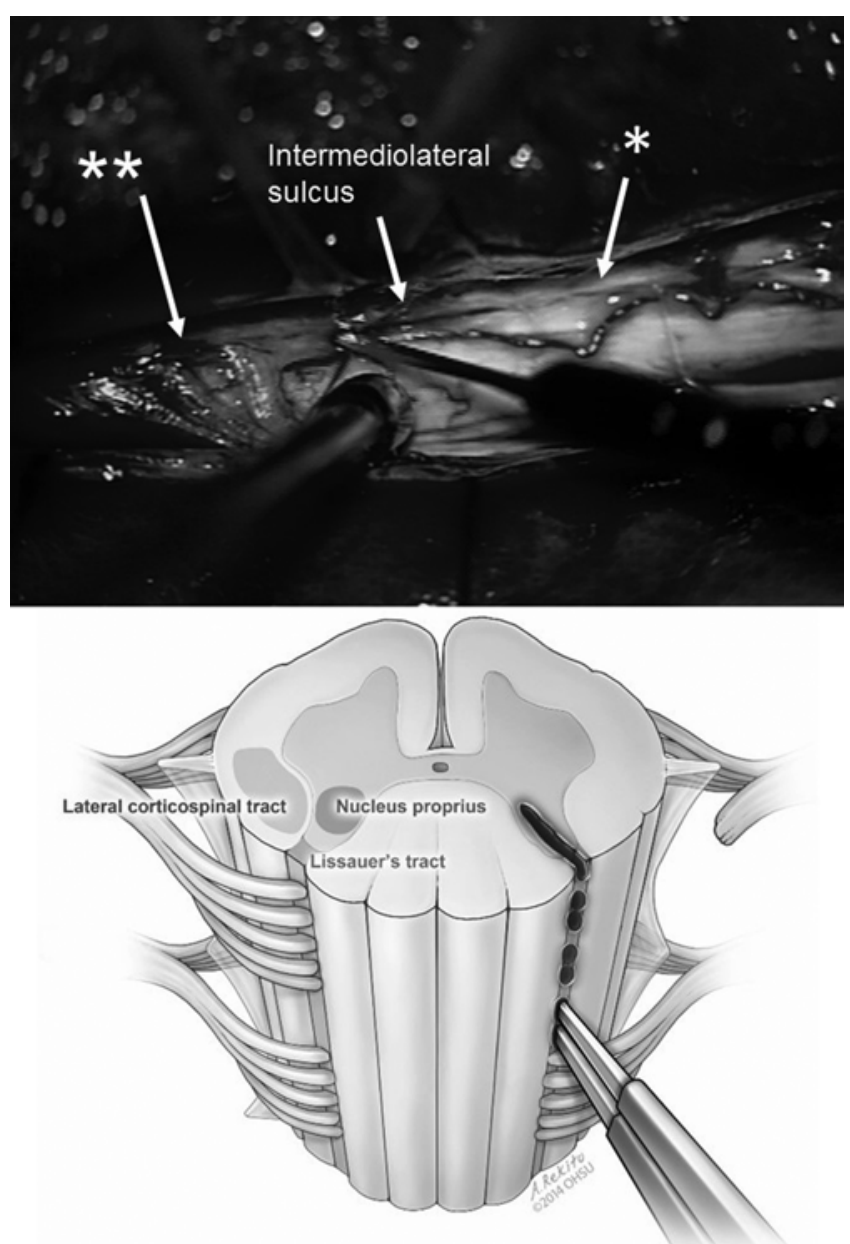

FIG. 1. Intraoperative photograph and schematic representation of DREZotomy. Upper: Intraoperative photograph depicting the incision into the intermediolateral sulcus of the spinal cord prior to lesion creation at the DREZ. Because hemilaminectomy was performed, the contralateral side is not visible; however, this technique preserves the spinous processes and midline tension band (top). Note the normal cervical rootlets on the left (double asterisks), which can be useful for localizing the DREZ when the normal anatomy is distorted by scarring. On the right, note the "pits" within the cord (single asterisk) that correlate with the sites of root avulsion. Lower: Schematic depiction of the staccato lesions created in the DREZ using microbipolar cautery. The target for lesion creation is the lateral portion of Lissauer's tract and the nucleus proprius to a depth of 2 to $3 \mathrm{~mm}$. Entry into the cord at a $45^{\circ}$ angle prevents damage to the adjacent lateral corticospinal tract. Lower panel: Copyright Andy Rekito. Published with permission.

\section{Imaging Assessment}

Preoperative cervical spine MR images were reviewed by 2 observers. All available MR images were examined for signs of nerve root avulsion, as exhibited by pseudomeningocele, intrinsic spinal cord signal change, or cord "pitting" where a portion of the spinal cord appears to have been avulsed along with the dorsal nerve root (Fig. 2). In particular, the intrinsic architecture of the spinal cord was examined for signs of damage to the dorsal horn or DREZ and categorized into 3 groups: normal, avulsion of the DREZ, or damage to the dorsal horn of the spinal cord.
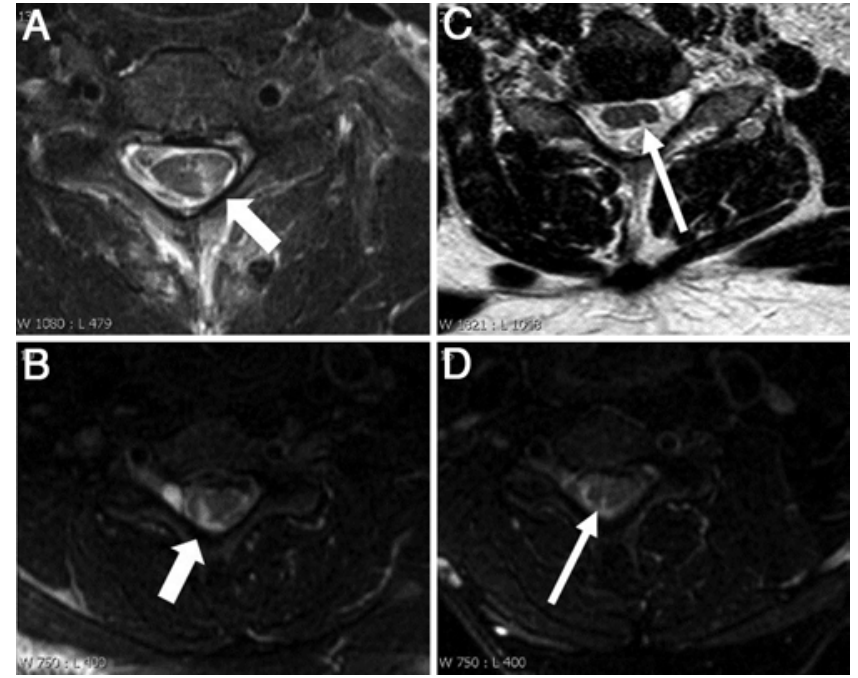

FIG. 2. MRI evidence of damage to the DREZ and dorsal horn after BPA. A and B: Damage caused by BPA to the dorsal horn is evidenced by T2 hyperintensity (thick arrows). C and D: Avulsion of the DREZ associated with cervical rootlet avulsion and subsequently evident by cord "pitting," which is denoted with thin arrows. Intrinsic spinal cord damage as evidenced by either of these 2 findings was found to be correlated with poorer outcomes after DREZotomy.

\section{Prognostic Factors}

The following patient- and pain-related factors were examined: age, sex, predominance of a lancinating or paroxysmal component of pain, duration of pain prior to surgery, and presence or absence of prior surgery on the brachial plexus or spinal cord. Imaging findings, as described above, were also examined, that is, intrinsic cord signal or evidence of mechanical damage to the spinal cord itself. Pseudomeningocele was not considered damage to the spinal cord unless accompanied by intrinsic cord signal or cord pitting.

\section{Statistical Analysis}

The statistical analysis was performed using commercially available software (MATLAB). Kaplan-Meier survival statistics were calculated and log-rank tests were performed. The Yates correction was applied to correct for the small sample size. Prognostic factors were analyzed using Cox proportional hazards modeling. Outcome values were compared using the Wilcoxon signed-rank or Student t-test, where appropriate, and correlations were calculated in the usual fashion. Contingency tables were constructed and statistics were calculated using chi-square statistics or Fisher exact test with or without the Freeman-Halton extension as appropriate. Multivariate analysis was performed using linear regression models. A full model using all predictors was constructed, followed by a forward and backward stepwise regression to obtain a more parsimonious model. Here, $\mathrm{p}$ values $<0.05$ were considered statistically significant.

\section{Results}

\section{Demographics and Clinical Characteristics}

Twenty-seven patients were identified as having un- 
dergone DREZotomy for neuropathic pain after BPA. The demographic data and clinical characteristics are summarized in Table 1 . The average age was 45.8 years (range 25.5-66.9 years). The vast majority of patients were male (96\%). The mechanisms of injury are presented in Table 1. A total of 9 patients had undergone elective shoulder disarticulation or revision of traumatic amputations. Four had some useful function, which consisted of at least antigravity strength at the biceps. The remaining 14 patients had minimal use of either the distal or proximal muscle groups. The median duration of pain prior to the procedure was 36 months (range 3-360 months). All patients had neuropathic pain, which was constant, and characterized as burning, aching, crushing, or cramping. In addition, 8 patients described a lancinating component, which consisted of brief stabbing pains. There were no significant differences between patients with imaging and those without imaging in terms of these characteristics (Table 1).

Follow-up was possible for all but 1 patient, as described above. The average follow-up was 108.0 months, and the median follow-up was 102.7 months. Patients who were operated on earlier were less likely to have MR imaging, and thus had longer follow-up times (mean 85 vs 136 months, respectively, $\mathrm{p}=0.03$; median 62.5 vs 159 months, respectively, $\mathrm{p}=0.03$ ).

\section{Pain Relief and Pain Recurrence After DREZ}

Overall, the immediate postoperative results included complete pain relief in 15 patients (55.6\%), 2 patients with more than $75 \%$ reduction in pain $(7.4 \%), 5$ patients with greater than $50 \%$ reduction of pain $(18.5 \%)$, and 5 patients $(18.5 \%)$ who experienced no significant change in their pain. Thus, the initial success rate of DREZotomy was $81.5 \%$. At the median follow-up time of 102.7 months, 17 patients maintained a good outcome (62.9\%). KaplanMeier survival statistics were calculated and are shown in Fig. 3. One patient experienced very late treatment failure with the return of constant burning pain at 77.0 months after surgery (Fig. 3). All other pain recurrences occurred within 12 months of surgery.

The treatment failure rates over time for patients with imaging were not significantly different from patients without imaging (log-rank test; $\mathrm{p}=0.44)$, and the median time to failure was not reached for patients with or without imaging. Initial success was obtained in 11 of $15(73.3 \%)$ patients, and at the median follow-up for the patients who underwent imaging (62.5 months) there was 1 treatment failure, resulting in a success rate of $66 \%$ at the median follow-up. One late treatment failure resulted in a final success rate of $53 \%$ (Fig. 3).

Of 27 patients who underwent DREZotomy for BPA, we were able to review the MRIs for 15 patients (55.6\%). The 2 observers independently reviewed the available images, and this resulted in complete agreement regarding the reported spinal cord abnormalities. Seven patients showed evidence of pseudomeningocele. Three patients had no intrinsic spinal cord abnormalities. Eleven patients showed T2 hyperintensity within the cord itself that extended into the DREZ. Five patients showed an avulsion in the spinal cord itself, or "pitting" at the DREZ (Fig. 2). Of these 5 patients, 4 showed both pitting at the DREZ and intrinsic T2 changes.

The Kaplan-Meier curves for the patients with normal and abnormal spinal cords did not significantly differ (logrank test; $p=0.63$ ). Median time to failure was not reached in either group. The presence or absence of lancinating pain did not affect the time to treatment failure (log-rank test; $p=0.52$ ). Previous surgery on the brachial plexus or spinal cord did not affect time to failure (log-rank test; $p$ $=0.22$ ). The duration of pain prior to DREZotomy was significantly correlated with increased time to failure $(\rho$ $=0.61 ; p=0.01$ ).

The Cox proportional hazards model that incorporated 2 prognostic factors - the duration of pain prior to surgery and the presence of abnormal findings on imaging-did not identify either factor as a significant predictor of treatment success over time (Table 2).

\section{Predictors of Treatment Success}

The outcome scores were significantly and negatively correlated with the degree of abnormality seen on imaging of the spinal cord (Fig. 3). An increase in the number of abnormalities (either intrinsic cord signal or DREZ avulsion) was correlated with a poorer outcome score $(\rho=-0.59$; $p=$ 0.02 ). The average outcome score in patients with an MRI showing a normal spinal cord was 3.67 versus an average score of 1.75 in patients with an avulsion or intrinsic cord signal (t-test; $\mathrm{p}=0.001$ ). All patients with MRI showing a normal spinal cord, with no intrinsic signal or damage to the DREZ, had excellent or pain-free outcomes. In contrast, only 2 of 12 patients with cord abnormalities on MRI had excellent relief from pain, and none were completely pain free (Table 3). The proportion of patients with poor, good, excellent, and pain-free outcomes is significantly different between patients with normal imaging and those showing intrinsic cord signals or evidence of avulsion of DREZ (Fisher exact test; $\mathrm{p}=0.009$ ).

Duration of pain prior to DREZotomy was significantly correlated with better outcomes (Fig. 3). Patients presenting with a longer history of pain had higher outcome scores $(\rho=0.70 ; p=0.004)$. The distribution of pain duration is not normal (Kolmogorov-Smirnov test; $p=0.00$ ), but similar results were obtained using ranked scores (Spearman rank correlation; $\rho=0.64 ; p=0.009$ ). Other predictors did not correlate with successful pain relief, and included the predominance of lancinating pain $(\rho=$ $-0.24 ; p=0.38$ ) and whether patients had undergone previous brachial plexus or spinal cord surgery $(\rho=-0.36$; $p$ $=0.17$ ). A linear regression model that incorporated these predictors was constructed, which showed significant predictive values for the duration of pain $(p=0.004)$ and abnormal imaging findings $(\mathrm{p}=0.024)$, but not for other factors (Table 4).

\section{Morbidity and Mortality}

No patient in this study died. There were no CSF leaks or infections in the study patients. No patient reported any gait ataxia or contralateral lower extremity weakness. Notably, no patient reported the worsening of pain after the DREZotomy procedure. 
TABLE 1. Demographics and clinical characteristics

\begin{tabular}{|c|c|c|c|}
\hline & $\begin{array}{c}\text { All } \\
\text { Patients* }\end{array}$ & $\begin{array}{l}\text { Imaged } \\
\text { Patients* }\end{array}$ & Statistics† \\
\hline \multicolumn{4}{|l|}{ Sex } \\
\hline Male & 26 & 15 & $\chi^{2}=0.57$ \\
\hline Female & 1 & 0 & $p=0.45$ \\
\hline \multicolumn{4}{|l|}{ Age, yrs } \\
\hline Mean & 45.8 & 46.9 & $p=0.55$ \\
\hline Median & 45.6 & 47.3 & $p=0.33$ \\
\hline Range & $25.5-66.9$ & $31.3-66.9$ & \\
\hline \multicolumn{4}{|l|}{ Laterality } \\
\hline Right & 15 & 8 & $\chi^{2}=0.2$ \\
\hline Left & 12 & 7 & $p=0.89$ \\
\hline \multicolumn{4}{|l|}{ Pain characteristics } \\
\hline Lancinating & 8 & 2 & $\chi^{2}=1.4$ \\
\hline Constant & 27 & 15 & $p=0.23$ \\
\hline \multicolumn{4}{|l|}{ Previous operations } \\
\hline Brachial plexus & 12 & 3 & \\
\hline Amputation & 9 & 4 & \\
\hline Spinal cord & 2 & 2 & $\chi^{2}=1.48$ \\
\hline Spinal cord stimulator & 4 & 2 & $p=0.48$ \\
\hline \multicolumn{4}{|l|}{$\begin{array}{l}\text { Preoperative duration of } \\
\text { pain, mos }\end{array}$} \\
\hline Mean & 91.0 & 107.0 & $p=0.35$ \\
\hline Median & 36 & 36 & $p=0.61$ \\
\hline Range & $3-360$ & $3-300$ & \\
\hline \multicolumn{4}{|l|}{ Follow-up duration, mos } \\
\hline Mean & 108.0 & 85.0 & $p=0.03$ \\
\hline Median & 102.7 & 62.5 & $p=0.03$ \\
\hline Range & $7.6-213.2$ & $7.6-197.1$ & \\
\hline \multicolumn{4}{|l|}{ Injury mechanism } \\
\hline Motor vehicle & 21 & 15 & \\
\hline Logging accident & 4 & 0 & \\
\hline Wood chipper & 1 & 0 & $\chi^{2}=3.89$ \\
\hline Crush injury & 1 & 0 & $p=0.27$ \\
\hline \multicolumn{4}{|l|}{ Residual function } \\
\hline Amputation & 9 & 4 & \\
\hline No useful function & 14 & 10 & $\chi^{2}=1.05$ \\
\hline Useful & 4 & 1 & $p=0.59$ \\
\hline \multicolumn{4}{|l|}{ Imaging } \\
\hline MRI & 15 & & \\
\hline No imaging & 12 & & \\
\hline \multicolumn{4}{|l|}{ Abnormality } \\
\hline Pseudomeningocele & 7 & & \\
\hline Cord signal & 11 & & \\
\hline Cord pitting & 5 & & \\
\hline Signal and pitting & 4 & & \\
\hline No cord abnormality & 3 & & \\
\hline \multicolumn{4}{|l|}{ Treated levels } \\
\hline Mean no. & 4.55 & & \\
\hline Median no. & 4 & & \\
\hline
\end{tabular}

TABLE 1. Demographics and clinical characteristics (continued)

\begin{tabular}{|c|c|c|c|}
\hline & $\begin{array}{c}\text { All } \\
\text { Patients* }\end{array}$ & $\begin{array}{l}\text { Imaged } \\
\text { Patients* }\end{array}$ & Statistics $†$ \\
\hline \multicolumn{4}{|c|}{ Treated levels (continued) } \\
\hline Range & $2-9$ & & \\
\hline Highest level & C-2 & & \\
\hline Lowest level & $\mathrm{T}-3$ & & \\
\hline
\end{tabular}

* Values are number of patients unless stated otherwise.

† Boldface values indicate statistical significance.

\section{Discussion}

The successful treatment of pain resulting from BPA with DREZotomy has been documented in many observational studies. A subset of studies have demonstrated lasting relief with an average follow-up of more than 5 years in more than 10 patients. ${ }^{1,2,5,6,12,23-25,29}$ There are only a few papers that report failure rates with meaningful actuarial data in the form of survival tables or Kaplan-Meier curves. ${ }^{1,9,23,27}$ None of these papers explicitly report the median follow-up time, and 2 papers report outcomes at the mean follow-up times. It is thus difficult to interpret these studies. Additionally, the outcome measures employed in these studies differ; however, in general, these studies consider a good outcome as a patient experiencing greater than $50 \%$ pain relief. Using this criterion, the reported rates of treatment success range from $41.9 \%$ to $76.5 \%$ (Table 5). Our study reports a success rate of $66 \%$ using this same standard, which is commensurate with what has been previously reported.

\section{Imaging and Pain Outcomes}

Previous studies have commented on alterations to the spinal cord due to brachial plexus injury.,27 Marked rotation or lateral deviation of the spinal cord was noted in $34.5 \%$ to $49 \%{ }^{27}$ of patients due to arachnoid adhesions. A significant proportion $(22.5 \%-36.4 \%)$ were also noted to have abnormal tissue near the dorsolateral sulcus on microscopic examination, which consisted of focal gliosis or microcystic cavitation within the DREZ or dorsal horn. ${ }^{1,27}$ There is evidence that the presence of lesions within the DREZ may play a role in pain generation after nerve root avulsion. ${ }^{10,16}$ Such lesions are often visible on imaging studies after BPA. ${ }^{4,17}$ However, such pathological, anatomical, or imaging findings have not been correlated with the clinical outcomes after DREZotomy.

In this study, the preoperative MR images of patients undergoing DREZotomy were examined for evidence of damage to the dorsal horn or DREZ. Hyperintense T2 signals within the spinal cord and involving the DREZ, or evidence of avulsion of the DREZ itself, were noted. The lack of such findings was significantly correlated with better outcomes by the univariate $(\mathrm{p}=0.02)$ and multivariate $(p=0.02)$ analyses. The average outcome score at the final follow-up was significantly higher in patients without evidence of DREZ or dorsal horn injury (3.67 vs 1.75 , t-test; $\mathrm{p}=0.001$ ). Importantly, the differences in final outcomes between these 2 groups is largely attributable to differences in the initial results of surgery rather than treatment 

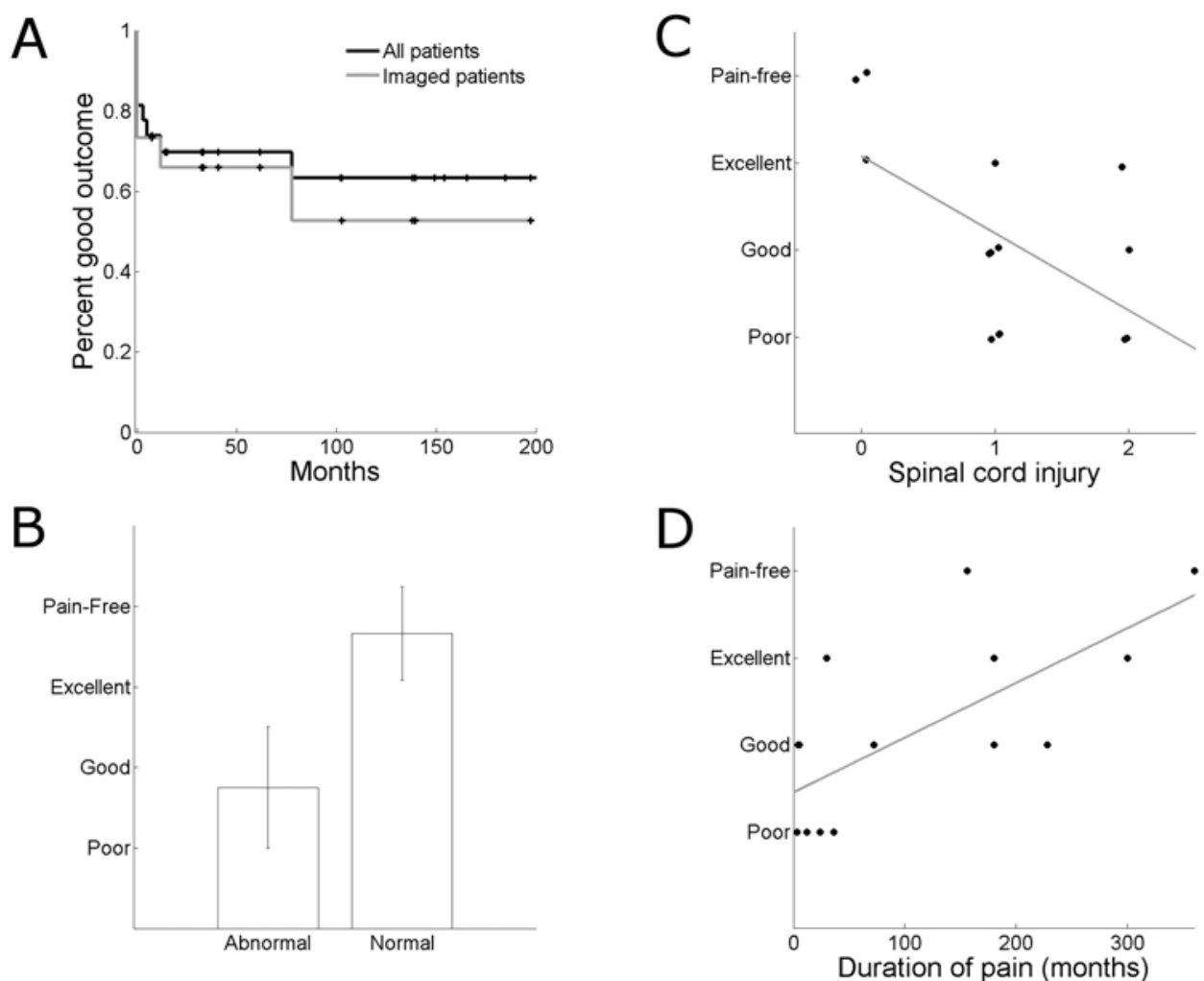

FIG. 3. Long-term results of DREZotomy. A: Kaplan-Meier curves for all patients treated with DREZotomy (black line) compared with patients treated with DREZotomy whose preoperative MR images were available for review (gray line). There was no significant difference in the time to treatment failure by the log-rank test $(p=0.44)$. B: Outcome scores for patients without MR evidence of injury to the spinal cord is significantly better than those exhibiting some abnormal signal or pitting in the cord. The average outcome score for the normal imaging patients was 3.67 compared with 1.75 in patients with abnormal imaging of the DREZ or dorsal horn (t-test; $p=0.001$ ). C: Outcomes are negatively correlated with an increasing degree of spinal cord injury: normal spinal cords (0), imaging showing either T2 signal within the dorsal horn or DREZ avulsion (1), and imaging showing both (2). The correlation is strong $(\rho=-0.587)$ and significant $(p=0.021)$. D: A longer duration of pain prior to DREZotomy is correlated with better outcomes $(\rho=0.70 ; p=0.003$ ). These findings were borne out in the multivariate analysis using a linear regression model that incorporated only these 2 predictors $(p=0.001)$.

failures over time; more patients had no initial response to surgery $(n=3)$ than experienced the return of pain after DREZotomy $(\mathrm{n}=2)$.

No patients with MRI abnormalities within the spinal cord had pain-free outcomes. The reasons for this may be related to the putative mechanism of pain generation after deafferentation. There is electrophysiological evidence of abnormal spontaneous and evoked activity in spinal interneurons after deafferentation ${ }^{14,16}$ and that DREZotomy normalizes this activity. ${ }^{10}$ It has been posited that unsuccessful treatment of deafferentation pain with destructive procedures within the spinal cord suggests a more proximal, central source of pain. ${ }^{28}$ Importantly, no patients in

TABLE 2. Cox proportional hazards model incorporating preoperative duration of pain and imaging findings

\begin{tabular}{lcccc}
\hline \multicolumn{1}{c}{ Predictors } & $\beta$ & $\begin{array}{c}\text { Hazard } \\
\text { Ratio }\end{array}$ & $\begin{array}{c}\text { Standard } \\
\text { Error }\end{array}$ & $\begin{array}{c}p \\
\text { Value }\end{array}$ \\
\hline Duration & -0.02 & 0.98 & 0.01 & 0.20 \\
\hline Abnormal cord & -1.79 & 0.17 & 2.41 & 0.46 \\
\hline
\end{tabular}

this study with normal MRI of the spinal cord had a poor outcome; that is, all patients with normal MRI had excellent or completely pain-free outcomes. This supports the notion that poor outcomes from DREZotomy may be related to the development of a central pain generator.

TABLE 3. Contingency table for outcomes and imaging findings*

\begin{tabular}{|c|c|c|c|}
\hline \multirow[b]{2}{*}{ Outcome } & \multicolumn{2}{|c|}{ MRI Findings } & \multirow[b]{2}{*}{ Total } \\
\hline & Normal Cord & Abnormal Cord & \\
\hline Poor & $0(0.4)$ & $5(4.0)$ & 5 \\
\hline Good & $0(0.6)$ & $5(4.0)$ & 5 \\
\hline Excellent & $1(1.0)$ & $2(2.4)$ & 3 \\
\hline Pain free & $2(1.0)$ & $0(1.6)$ & 2 \\
\hline Total & 3 & 12 & 15 \\
\hline Chi-square test & $\chi^{2}=10.83$ & $d f=3$ & $p=0.013$ \\
\hline Fisher exact test & $p=0.009$ & & \\
\hline
\end{tabular}

* Expected values based on the chi-square distribution are shown in parentheses. 
TABLE 4. Linear regression model for the predictors of treatment outcome

\begin{tabular}{|c|c|c|c|c|}
\hline \multirow{2}{*}{$\begin{array}{c}\text { Correlations } \\
\text { Predictors }\end{array}$} & \multicolumn{4}{|c|}{ Value } \\
\hline & Lancinating & Duration & Previous & Abnormal MR \\
\hline \multicolumn{5}{|l|}{ Lancinating } \\
\hline Duration & -0.184 & & & \\
\hline Previous & -0.277 & $-0.548^{*}$ & & \\
\hline Abnormal MR & -0.039 & -0.247 & 0.347 & \\
\hline Outcome & -0.243 & $0.700^{*}$ & -0.367 & $-0.587^{*}$ \\
\hline Full model & Coefficient & Standard error & p Value & \\
\hline Constant & 1.277 & 0.506 & 0.030 & \\
\hline Lancinating & -0.360 & 0.597 & 0.560 & \\
\hline Duration & 0.0055 & 0.002 & 0.022 & \\
\hline Previous & 0.219 & 0.522 & 0.683 & \\
\hline Abnormal MR & -0.715 & 0.278 & 0.028 & \\
\hline RMS error & 0.685 & & & \\
\hline $\mathrm{R}^{2}$ & 0.582 & & & \\
\hline $\mathrm{F}$ & 5.87 & & & \\
\hline$p$ value & 0.010 & & & \\
\hline Pruned model & Coefficient & Standard error & p Value & \\
\hline Constant & 1.273 & 0.391 & 0.007 & \\
\hline Duration & 0.005 & 0.002 & 0.005 & \\
\hline Abnormal MR & -0.665 & 0.257 & 0.024 & \\
\hline RMS error & 0.655 & & & \\
\hline$R^{2}$ & 0.618 & & & \\
\hline $\mathrm{F}$ & 12.3 & & & \\
\hline$p$ value & 0.001 & & & \\
\hline
\end{tabular}

Alternatively, the poorer outcomes in patients with abnormal spinal cords on MRI may indicate that larger DREZ lesions should have been made during surgery. Cord signal change or pitting may be biomarkers of a more severe initial injury that necessitates a larger lesion in order to encompass the aberrant pain generators. This must be considered in light of the increasing complication rates with larger lesions. In 1 study, ultrasound was used to map the depth of the microcavities within the dorsal horn and tailor lesions to ablate all abnormal tissue. ${ }^{6}$ This resulted in $100 \%$ initial pain relief in 107 patients and long-term, good pain relief in $87 \%$ of patients at an average followup of 47.5 months. ${ }^{6}$ It is difficult to determine if this is a significantly improved success rate over the methods used in this paper; the rate of ataxia and lower-extremity weakness was certainly higher at $17 \% .^{6}$

Our contention is that in cases where damage to the dorsal horn and DREZ is evident on MRI, deafferentation pain is more likely to be the result of altered activity in thalamocortical circuitry rather than hyperactivity within the somatosensory or spinothalamic pathways.

\section{Other Prognostic Factors}

Several other prognostic factors for successful pain relief after DREZotomy have been described in previous

TABLE 5. Reports in the literature

\begin{tabular}{lcccc}
\hline \multicolumn{1}{c}{ Authors \& Year } & $\begin{array}{c}\text { No. of } \\
\text { Patients }\end{array}$ & $\begin{array}{c}\text { Mean Follow-Up, } \\
\text { Yrs (range) }\end{array}$ & $\begin{array}{c}\text { Percentage of Good Results } \\
\text { at Last Follow-Up }\end{array}$ & $\begin{array}{c}\text { Percentage of Good Results } \\
\text { at Mean Follow-Up }\end{array}$ \\
\hline Friedman et al., 1988 & 39 & $4.7(1.1-10)$ & $67 \%$ & NR \\
\hline Sindou et al., 2005 & 44 & $6.0(1.0-12)$ & $65.9 \%$ & $59.8 \%$ \\
\hline Aichaoui et al., 2011 & 29 & $5.0(1.0-10.2)$ & $41.9 \%$ & $60 \%$ \\
\hline Prestor et al., 2006 & 26 & NR (NR) & $76.5 \%$ & NR \\
\hline Chen et al., 2006 & 40 & NR (5.0-18.0) & $60 \%$ & NR \\
\hline NR = not reported. & & & &
\end{tabular}


studies. A lengthier preoperative duration of pain has been correlated with better long-term results from DREZotomy. ${ }^{23}$ The pain characteristics have also been advanced as predictive factors; relief from paroxysmal pain is more likely than relief from constant pain. ${ }^{1}$

In this study, the duration of pain prior to DREZotomy was significantly correlated with a better outcome (Spearman's $\rho=0.64 ; p=0.009)$. All patients with poor outcomes underwent DREZotomy within 36 months of onset of post-BPA pain. This finding was borne out in the multivariable analysis, with the linear regression model finding that duration of pain was a significant predictor of outcome score $(p=0.004)$. Interestingly, the duration of pain was also negatively correlated with previous brachial plexus or spinal cord surgery $(\rho=-0.548 ; p=0.034)$; in other words, patients with a long preoperative pain duration were significantly less likely to have undergone a previous surgery to restore function or address their pain. It is possible that those seeking multiple treatments at a relatively early time after onset of pain represent a distinct subset of patients with refractory disease.

Perhaps surprisingly, the presence or absence of lancinating pain was not a predictor of outcomes in the univariate or multivariate analysis. Previous studies have found an absolute increase of $11.5 \%$ in good outcomes when treating paroxysmal pain using DREZotomy. ${ }^{1}$ Similar results have been obtained for other destructive procedures used to treat pain with a spinal cord origin. ${ }^{28}$ There are several possible explanations for this discrepancy. In our study, only 2 of 15 patients presented with lancinating pain as the major pain component. Additionally, in this study, only global pain was assessed as an outcome, and it is not known if a specific improvement in paroxysmal pain was experienced.

\section{Study Weaknesses}

This is a small, retrospective study from a single institution. It therefore suffers from the same potential sources of bias present in all observational studies. There was no objective preoperative pain assessment, and thus reports of preoperative pain scores are subject to recall bias. That said, there was little variation in patient responses, as the preoperative pain levels were uniformly reported as 9 or 10 of 10 on VAS. Of the 27 patients who underwent DREZotomy, only 15 underwent MRI of the spine prior to their procedure. Thus, the presence of selection bias cannot be ignored. We did not, however, see any differences in the prognostic factors that we examined between patients with imaging and without imaging (Table 1). Observational bias was mitigated by using an interviewer not directly involved in patient care and having imaging reviewed by 2 independent observers. Response bias was minimal in this study, as the loss to follow-up was quite low, with only 1 patient censored at 3 months. The mean and median follow-up were quite long at 85.0 and 62.5 months, respectively.

Despite controlling for some sources of bias, the small sample size in this study remains a concern. For the univariate tests, the Fisher exact test was used due to the small sample size. Predictors for the Cox proportional hazards model were limited to 2 factors. However, the accuracy of the statistical modeling would obviously be increased with a greater number of patients in the study. And, again, the effects of recall and selection bias cannot be ignored.

Like all reports on the effectiveness of DREZotomy for BPA pain, there is no control group for comparing outcomes. This is particularly relevant given that the natural history of BPA pain is largely undetermined. One longterm study of patients with BPA who did not undergo surgical treatment reports that after 4 years, $25 \%$ of patients continued to have severe pain that was considered unacceptable, and after 11 years this number dropped to $17 \% .^{20}$ However, within this cohort of patients, only $6 \%$ had spontaneous and complete remission of pain. ${ }^{20}$ It seems that the natural history of BPA pain is unremitting pain, but this becomes tolerable in a majority of patients; whether the rate of acceptable pain levels after surgical intervention is better than the rate of $75 \%$ after 4 years achieved by conservative observation is an open question.

Further work is necessary to address these questions. Certainly, correlating imaging findings with outcomes in a larger cohort of patients is warranted. Given the mechanism of cervical root avulsion, it may seem surprising that any patients lack evidence of damage to the DREZ or dorsal horn. High-field MRI may provide a more granular examination of the imaging findings and be helpful for prognostication; advanced imaging, such as DTI of the spinal cord itself, or imaging evidence of a central pain generator within the brain itself, may likewise provide more information regarding who may benefit from this procedure. Finally, comparison with a control group would also be useful.

\section{Conclusions}

DREZotomy is a safe, effective, and durable treatment for pain resulting from BPA. This study examined the long-term results of this procedure for intractable pain and finds that three-quarters of patients have immediate and meaningful relief of pain, and overall two-thirds of patients maintained this result for longer than 5 years. Significantly, no patients reported an increase in pain over time. These results are in contrast to many ablative procedures for pain that have transient effects and can result in the worsening of deafferentation pain.

Preoperative MRI may have prognostic value, as all patients who do not show damage to the DREZ or dorsal horn have good outcomes throughout the duration of follow-up, which ranged from 7.6 months to 197.1 months. Interestingly, duration of pain prior to DREZotomy was also correlated with good outcomes. These results may provide important prognostic information and deserve further investigation in a larger, prospective study.

\section{References}

1. Aichaoui F, Mertens P, Sindou M: Dorsal root entry zone lesioning for pain after brachial plexus avulsion: results with special emphasis on differential effects on the paroxysmal versus the continuous components. A prospective study in a 29-patient consecutive series. Pain 152:1923-1930, 2011

2. Awad AJ, Forbes JA, Jermakowicz W, Eli IM, Blumenkopf B, Konrad P: Experience with 25 years of dorsal root entry zone lesioning at a single institution. Surg Neurol Int 4:64, 2013

3. Bruxelle J, Travers V, Thiebaut JB: Occurrence and treatment 
of pain after brachial plexus injury. Clin Orthop Relat Res (237):87-95, 1988

4. Carvalho GA, Nikkhah G, Matthies C, Penkert G, Samii M: Diagnosis of root avulsions in traumatic brachial plexus injuries: value of computerized tomography myelography and magnetic resonance imaging. J Neurosurg 86:69-76, 1997

5. Chen HJ, Tu YK: Long term follow-up results of dorsal root entry zone lesions for intractable pain after brachial plexus avulsion injuries. Acta Neurochir Suppl 99:73-75, 2006

6. Dreval ON: Ultrasonic DREZ-operations for treatment of pain due to brachial plexus avulsion. Acta Neurochir (Wien) 122:76-81, 1993

7. Falci S, Best L, Bayles R, Lammertse D, Starnes C: Dorsal root entry zone microcoagulation for spinal cord injuryrelated central pain: operative intramedullary electrophysiological guidance and clinical outcome. J Neurosurg 97 (2 Suppl):193-200, 2002

8. Friedman AH, Bullitt E: Dorsal root entry zone lesions in the treatment of pain following brachial plexus avulsion, spinal cord injury and herpes zoster. Appl Neurophysiol 51:164169,1988

9. Friedman AH, Nashold BS Jr, Bronec PR: Dorsal root entry zone lesions for the treatment of brachial plexus avulsion injuries: a follow-up study. Neurosurgery 22:369-373, 1988

10. Guenot M, Bullier J, Sindou M: Clinical and electrophysiological expression of deafferentation pain alleviated by dorsal root entry zone lesions in rats. J Neurosurg 97:1402-1409, 2002

11. Jeanmonod D, Sindou M, Mauguière F: Intraoperative electrophysiological recordings during microsurgical DREZtomies in man. Stereotact Funct Neurosurg 54-55:80-85, 1990

12. Kanpolat Y, Tuna H, Bozkurt M, Elhan AH: Spinal and nucleus caudalis dorsal root entry zone operations for chronic pain. Neurosurgery 62 (3 Suppl 1):235-244, 2008

13. Levy WJ, Nutkiewicz A, Ditmore QM, Watts C: Laser-induced dorsal root entry zone lesions for pain control. Report of three cases. J Neurosurg 59:884-886, 1983

14. Loeser JD, Ward AA Jr: Some effects of deafferentation on neurons of the cat spinal cord. Arch Neurol 17:629-636, 1967

15. Malin JP, Winkelmüller W: Phantom phenomena (phantom arm) following cervical root avulsion. Effect of dorsal root entry zone thermocoagulation. Eur Arch Psychiatry Neurol Sci 235:53-56, 1985

16. Mertens P, Ghaemmaghami C, Bert L, Perret-Liaudet A, Guenot M, Naous H, et al: Microdialysis study of amino acid neurotransmitters in the spinal dorsal horn of patients undergoing microsurgical dorsal root entry zone lesioning. Technical note. J Neurosurg 94 (1 Suppl):165-173, 2001

17. Mertens P, Guenot M, Hermier M, Jouvet A, Tournut P, Froment JL, et al: Radiologic anatomy of the spinal dorsal horn at the cervical level (anatomic-MRI correlations). Surg Radiol Anat 22:81-88, 2000

18. Midha R: Epidemiology of brachial plexus injuries in a multitrauma population. Neurosurg 40:1182-1189, 1997

19. Nashold BS Jr, Ostdahl RH: Dorsal root entry zone lesions for pain relief. J Neurosurg 51:59-69, 1979

20. Parry CB: Pain in avulsion lesions of the brachial plexus. Pain 9:41-53, 1980

21. Pereira EAC, Boccard SG, Linhares P, Chamadoira C, Rosas
MJ, Abreu P, et al: Thalamic deep brain stimulation for neuropathic pain after amputation or brachial plexus avulsion. Neurosurg Focus 35(3):E7, 2013

22. Piva B, Shaladi A, Saltari R, Phd GG: Spinal cord stimulation in the management of pain from brachial plexus avulsion. Neuromodulation 6:27-31, 2003

23. Prestor B: Microcoagulation of junctional dorsal root entry zone is effective treatment of brachial plexus avulsion pain: long-term follow-up study. Croat Med J 47:271-278, 2006

24. Prestor B: Microsurgical junctional DREZ coagulation for treatment of deafferentation pain syndromes. Surg Neurol 56:259-265, 2001

25. Samii M, Bear-Henney S, Lüdemann W, Tatagiba M, Blömer $\mathrm{U}$ : Treatment of refractory pain after brachial plexus avulsion with dorsal root entry zone lesions. Neurosurgery 48:12691277, 2001

26. Sindou M: Microsurgical DREZotomy (MDT) for pain, spasticity, and hyperactive bladder: a 20 -year experience. Acta Neurochir (Wien) 137:1-5, 1995

27. Sindou MP, Blondet E, Emery E, Mertens P: Microsurgical lesioning in the dorsal root entry zone for pain due to brachial plexus avulsion: a prospective series of 55 patients. $\mathbf{J}$ Neurosurg 102:1018-1028, 2005

28. Tasker RR, DeCarvalho GT, Dolan EJ: Intractable pain of spinal cord origin: clinical features and implications for surgery. J Neurosurg 77:373-378, 1992

29. Thomas DG, Kitchen ND: Long-term follow up of dorsal root entry zone lesions in brachial plexus avulsion. J Neurol Neurosurg Psychiatry 57:737-738, 1994

30. Wellons JC, Gorecki JP, Friedman AH: Stump, phantom and avulsion pain, in Burchiel KJ (ed): Surgical Management of Pain. New York: Thieme Medical Publishers, 2002

\section{Disclosure}

The authors report no conflict of interest concerning the materials or methods used in this study or the findings specified in this paper.

\section{Author Contributions}

Conception and design: Ko, Burchiel. Acquisition of data: Ko, Ozpinar, Magill, Raslan. Analysis and interpretation of data: Ko. Drafting the article: Ko, Raskin. Critically revising the article: Ko, Burchiel. Reviewed submitted version of manuscript: Ko, Raskin, Raslan, Burchiel. Approved the final version of the manuscript on behalf of all authors: Ko. Statistical analysis: Ko. Administrative/technical/material support: Burchiel. Study supervision: Burchiel.

\section{Supplemental Information}

Current Affiliation

Dr. Ozpinar: Department of Neurological Surgery, University of Pittsburgh Medical Center, Pittsburgh, Pennsylvania.

\section{Correspondence}

Andrew L. Ko, Department of Neurological Surgery, University of Washington, 1959 NE Pacific St., Seattle, WA 98195. email: alko00@u.washington.edu. 